\title{
Phase synchronization and topological defects in inhomogeneous media
}

\author{
Jörn Davidsen 7 and Raymond Kapral \\ Chemical Physics Theory Group, Department of Chemistry, \\ University of Toronto, Toronto, ON M5S 3H6, Canada
}

(Dated: October 23, 2018)

\begin{abstract}
The influence of topological defects on phase synchronization and phase coherence in twodimensional arrays of locally-coupled, nonidentical, chaotic oscillators is investigated. The motion of topological defects leads to a breakdown of phase synchronization in the vicinities of the defects; however, the system is much more phase coherent as long as the coupling between the oscillators is strong enough to prohibit the continuous dynamical creation and annihilation of defects. The generic occurrence of topological defects in two and higher dimensions implies that the concept of phase synchronization has to be modified for these systems.

PACS numbers: 05.45.Xt, 05.40.-a
\end{abstract}

The rich collective behavior, including mutual entrainment and self-synchronization, seen in systems of coupled oscillators has been a stimulus for the long-standing interest in these systems (see, for example, Refs. [1, 2, 3] and references therein). Recently, attention has turned to the study of coupled chaotic oscillators and, in particular, to the phenomenon of phase synchronization. Provided that the phase can be defined [4, 何, two coupled nonidentical chaotic oscillators are said to be phase synchronized if their frequencies are locked [1, 6]. This appears to be a general phenomenon and it has been observed in such diverse systems as electrically coupled neurons [7, 8], biomedical systems [9], chemical systems [10], and spatially extended ecological systems [11], to name only a few. Moreover, the potential role of phase synchronization in brain functions has been recognized [12, 13].

Much of the theoretical analyses of phase synchronization have been carried out on systems consisting of two locally coupled oscillators [6] or many globally coupled oscillators [14]. Large one-dimensional chains of locally coupled chaotic oscillators have been investigated very recently 15, 16, 17]. Here, we address the question whether phase synchronization can persist in higher spatial dimensions where topological defects can play a central role. We show that the existence of topological defects can lead to a breakdown of global phase synchronization in two-dimensional arrays of nonidentical chaotic oscillators. While most of the medium may remain phase synchronized, oscillators close to moving topological defects have a different frequency. Despite this fact, the phase coherence of the system is higher than in systems without topological defects. The transition to phase synchronization via phase clustering observed in one-dimensional systems 15,18 is not found in our simulations on twodimensional systems; instead, a transition involving point defects occurs.

Point topological defects in two-dimensional homoge-

\footnotetext{
*ioern.davidsen@utoronto.ca

†rkapral@chem.utoronto.ca
}

neous oscillatory media are associated with the appearance of spiral waves [19, 20, 21]. The phase field $\phi(\mathbf{r}, t)$ of a medium with a spiral wave contains a point topological phase defect in the spiral core such that

$$
\frac{1}{2 \pi} \oint \nabla \phi(\mathbf{r}, t) \cdot d \mathbf{l}= \pm n_{t},
$$

where $n_{t}$ is the topological charge of the defect [22]. A topological defect corresponds to a point in the medium where the local amplitude is zero and the phase is not defined. For periodic boundary conditions, the net topological charge of the medium is zero. For identical chaotic oscillators [23], spatially coherent spiral dynamics can still exist implying a phase locking of the oscillations [24.

To illustrate the phase synchronization properties in two-dimensional networks of nonidentical chaotic oscillators, we consider an $L \times L$ array of locally coupled Rössler oscillators with periodic boundary conditions [25],

$$
\frac{\partial \mathbf{x}(\mathbf{r}, t)}{\partial t}=\mathbf{R}(\mathbf{x}(\mathbf{r}, t))+K \sum_{\hat{\mathbf{r}} \in N(\mathbf{r})}(\mathbf{x}(\hat{\mathbf{r}}, t)-\mathbf{x}(\mathbf{r}, t)),
$$

where $R_{1}=-\omega(\mathbf{r}) x_{2}-x_{3}, R_{2}=\omega(\mathbf{r}) x_{1}+0.2 x_{2}, R_{3}=$ $x_{1} x_{3}-5.9 x_{3}+0.2$. The sites of the lattice are labelled by $\mathbf{r}, K$ is the coupling constant and $N(\mathbf{r})$ is the set of the four next nearest neighbors of site $\mathbf{r}$. The phase angles of the oscillators are given by $\phi(\mathbf{r}, t)=\arctan \frac{x_{2}(\mathbf{r}, t)}{x_{1}(\mathbf{r}, t)}$. To obtain the results presented below, we take $L=64$ and choose the $\omega(\mathbf{r})$ 's randomly from a uniform distribution on the interval $[0.95,1.05]$, ensuring that the system is in the chaotic regime.

First, we consider $K=K_{0} \equiv 0.1$. For different initial conditions leading to stable patterns, basically two scenarios can be observed. For homogeneous initial conditions or initial conditions with small inhomogeneities, no topological defects are created and the system evolves to a target pattern similar to the one in Fig. 1 (left panel). In the case of larger inhomogeneities in the initial conditions, a number of topological defects with $n_{t}= \pm 1$ is created initially. Some pairs of topological defects with opposite charge are quickly annihilated until only a small 

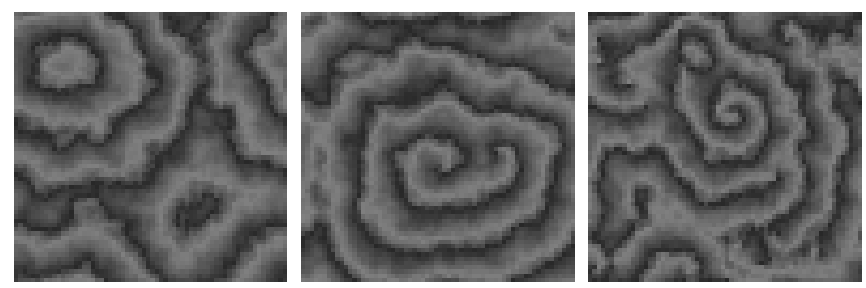

FIG. 1: Snapshots of the phase field, all for the same realization of $\omega(\mathbf{r})$. From left to right: $K=0.05$ without topological defects, $K=0.05$ with two surviving topological defects, and $K=0.0419$. In the latter case, topological defects are generated dynamically in pairs such that the number of topological defects fluctuates around a value of 8 pairs.

number is left generating a spiral pattern similar to that in Fig. 1 (middle panel). The topological defects are not necessarily stationary and for very long times it is possible that all defects could disappear through further annihilation events; however, the motion of the surviving topological defects is very slow compared to the initial annihilation processes and no further annihilation events were observed on our long simulation time scale $\left(10^{4}-10^{5}\right.$ spiral revolutions). These two scenarios persist provided $K$ is not too small.

When there are no topological defects, the system is phase synchronized. The occurrence of phase synchronization for coupled Rössler oscillators is usually attributed to the high degree of phase coherence of the attractor of a single Rössler oscillator [1] although this might not be a sufficient condition [5]. Phase coherence means that $\left(\int_{-\infty}^{\infty} d \tau\langle\eta(t) \eta(t+\tau)\rangle_{t}\right)^{1 / 2} \ll \omega_{0}$, where $d \tilde{\phi} / d t=\omega_{0}+\eta(t), \tilde{\phi}$ is the unbounded phase and $\langle\cdots\rangle_{t}$ signifies an average over $t$. If $\eta$ is $\delta$-correlated Gaussian noise this condition reduces to $\sigma \ll \omega_{0}$ and corresponds to a very narrow peak of width $\sigma^{2}$ at $\omega_{0}$ in the power spectrum of $x_{1}(t)$. However, generally temporal correlations in $\eta$ exist. These correlations determine the speed of convergence of the time average $\bar{\omega}(T)=T^{-1}(\tilde{\phi}(T)-\tilde{\phi}(0))$ towards $\omega_{0}$. For the system of coupled Rössler oscillators considered here, the speed of convergence - as measured by the standard deviation of the ensemble distribution of $\bar{\omega}(T)$ - scales as $1 / T$ as shown in Fig. 2. This is in contrast to what one would expect if the $\eta$ variables were independent where one obtains $1 / \sqrt{T}$ scaling since $\bar{\omega}(T)-\omega_{0}=1 / T \int_{0}^{T} d t \eta(t)$ and the standard deviation of $\int_{0}^{T} d t \eta(t)$ scales with $\sqrt{T}$. The observed variation with $T$ is much faster, implying an extremely high degree of phase coherence, even in the coupled system. The origin of this effect resides in the shape of the local attractors. They are two-banded with distinct frequencies $\omega_{1}$ and $\omega_{2}$ such that $\omega_{0}=\left(\omega_{1}+\omega_{2}\right) / 2$. Moreover, each deviation from $\omega_{1}$ is followed by a deviation from $\omega_{2}$ of similar amplitude but with opposite sign, and vice versa. Hence,

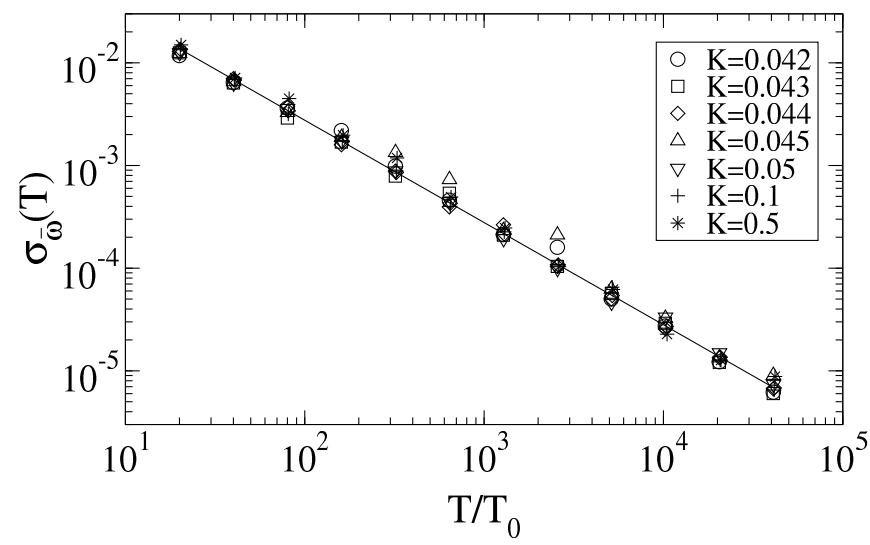

FIG. 2: Log-log plot of the standard deviation $\sigma_{\bar{\omega}}(T)$ of the $\bar{\omega}(T)$ distribution in the phase synchronized state describing the speed of convergence towards $\omega_{0}$. The solid line with slope -1 is plotted to guide the eye. Note that $T_{0}=2 \pi / \omega_{0}$ depends slightly on $K$.

the deviations of $\bar{\omega}(T)$ from $\omega_{0}$ should be dominated by the switching between the bands for not too large values of $T$. Thus, the maximal deviation from $\omega_{0}$ is given by the term $\left(\omega_{1}-\omega_{2}\right) /(2 T)$, reproducing the observed scaling. The two-banded structure can also be found in the ensemble distribution of $\bar{\omega}(T)$. There, two distinct peaks can be seen for certain $T$ values consistent with the fluctuations around the $1 / T$ scaling in Fig. 2.

If topological defects are present global phase synchronization is no longer guaranteed. Since a topological defect corresponds to a point in the system where a phase variable cannot be defined, this implies that the concept of phase synchronization can only be applied to parts of the system which exclude topological defects. For discrete lattices of oscillators considered here, this does not pose any problems because the defect is located almost surely between lattice sites. Thus, the presence of topological defects does not exclude global phase synchronization a priori. We indeed observe phase synchronization for quasi-stationary topological defects which explains the occurrence of phase synchronization in the presence of topological defects described in Ref. [26]. However, the motion of topological defects destroys phase synchronization locally. As shown in Fig. 3, the motion of a topological defect leads to a distortion of nearby local orbits. This in turn influences their local frequencies such that the system is no longer phase synchronized as can be deduced from the corresponding $\omega_{0}$ distribution in Fig. 1 [27. In particular, the "outliners" in this distribution are located in the vicinity of the topological defect(s).

For homogeneous oscillatory media it is well known that the time scales for the motions of topological defects depend on the system parameters, ranging from very slow motion to rapid dynamics 28]. Inhomogeneity 


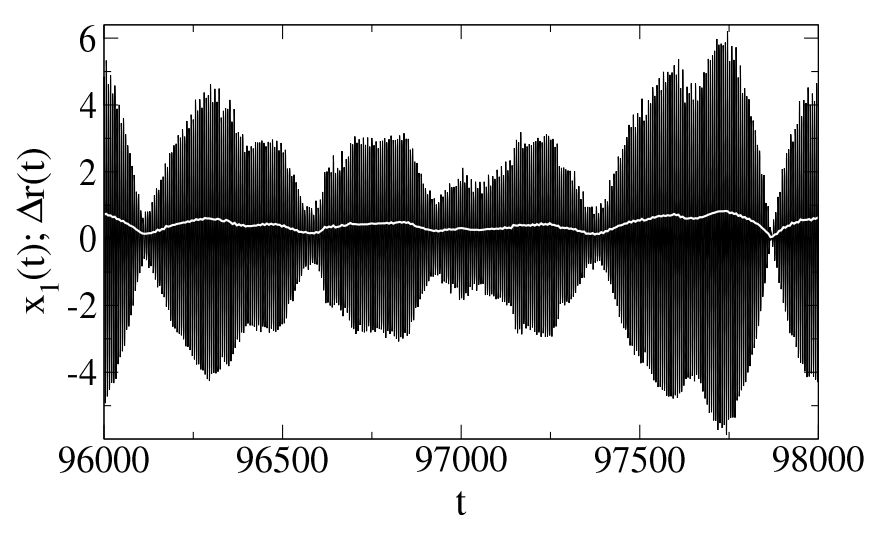

FIG. 3: Trajectory of $x_{1}(t)$ (black) for a fixed oscillator and the distance $\Delta r(t)$ (white) of this oscillator from the moving topological defect for $K=0.06$. The two temporal behaviors are correlated, demonstrating the influence of the topological defect's motion on the local orbit.

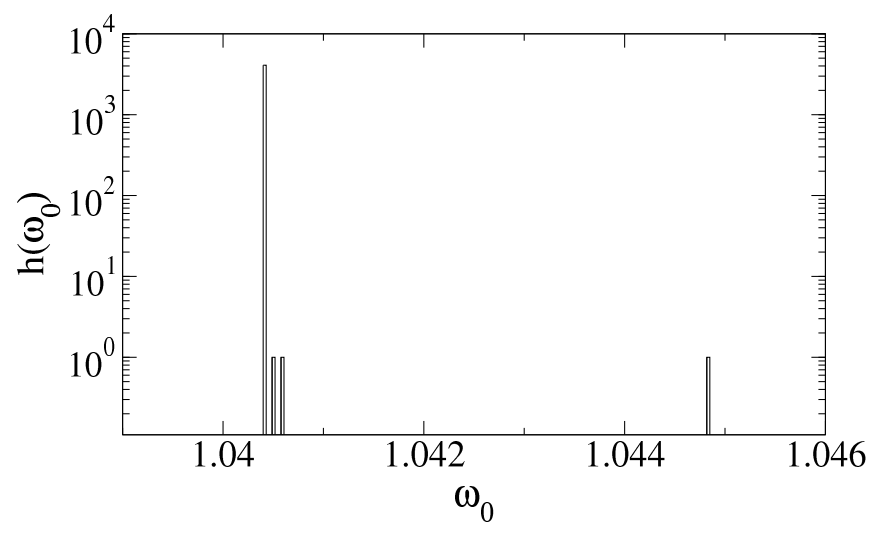

FIG. 4: The $\omega_{0}$ histogram $h\left(\omega_{0}\right)$ of the oscillators for $K=0.06$ with two surviving topological defects in the system. One defect moves causing the breakup of global phase synchronization. The exact shape of the distribution depends on the $\omega(\mathbf{r})$ realization. Negative and/or positive "ouliners" can exist and correspond to oscillators in the vicinity of the moving topological defect.

provides another mechanism for defect motion and, in the present case of a network of non-identical Rössler oscillators, this is the dominant mechanism. For a network of identical Rössler oscillators, the topological defects are quasi-stationary on the time scales considered here; thus, the observed breakup of global phase synchronization for this system is due to the quenched disorder in the network.

Although global phase synchronization is lost in the presence of moving topological defects, the fluctua- tions of the instantaneous period in the medium are greatly reduced. While the phase coherence criterion, $\left(\int_{-\infty}^{\infty} d \tau\langle\eta(t) \eta(t+\tau)\rangle_{t}\right)^{1 / 2} \ll \omega_{0}$, is satisfied to a high degree of accuracy as in the case without topological defects, the average amplitude of the fluctuations in the instantaneous periods $T(\mathbf{r}, n)=2 \pi / \omega(\mathbf{r}, n)$ as measured by $S \equiv\left\langle\langle S(\mathbf{r})\rangle_{\mathbf{r}}\right\rangle_{\omega}$ with

$$
S(\mathbf{r})=\frac{\langle T(\mathbf{r}, n)\rangle_{n}}{\sqrt{\left\langle\left(T(\mathbf{r}, n)-\langle T(\mathbf{r}, n)\rangle_{n}\right)^{2}\right\rangle_{n}}},
$$

are significantly different. Here, $T(\mathbf{r}, n)$ is the time needed for the $n$th rotation at site $\mathbf{r}$ and $\langle\cdots\rangle_{\omega}$ is the average over all realizations of $\omega(\mathbf{r})$. Figure 5 (left panel) shows that the value of $S$ is much larger above a certain coupling $K_{c}$ when (more) topological defects are present in the system, corresponding to a higher degree of coherence. An analysis of $S(\mathbf{r})$ shows that high values exist, especially in the vicinity of quasi-stationary topological defects (see right panel of Fig. 5). This is due to the influence of topological defects on the shapes of the local orbits: they are close to periodic limit cycles. This is in accord with observations in 29] for identical oscillators although the "effective" spatial period doubling cascade found there is absent in the present case.

The system maintains memory of its initial condition above $K_{c} \approx 0.043$ leading to the different values of $S$. For single $\omega(\mathbf{r})$ realizations, different scenarios, with either no defects or with defects present, can be observed for $K_{0}>K>K_{c}$ 30]. For $K$ below $K_{c}$, however, a qualitatively new behavior occurs. Topological defects are now generated and annihilated continuously by the dynamics which is responsible for the loss of memory of the initial condition. The number of topological defects fluctuates around a mean value which increases with decreasing $K$. This is expected because $K$ should control the characteristic length scale in the system. Moreover, the topological defects move on average much faster than above $K_{c}$, similar to defect mediated turbulence 31. The creation and annihilation of topological defects as well as the fast motion lead to strong distortion of the local orbits affected by the moving defects. This makes it impossible to define a proper phase variable for most of the oscillators in the network. Consequently, the concept of phase synchronization can no longer be applied.

In Refs. [15, 18], the transition to phase synchronization via the route of phase clustering and merging of clusters was investigated for chains of oscillators. Liu, et al. 115] concluded that phase clustering should be more prevalent than full phase synchronization, especially in networks of coupled neurons. However, the twodimensional system considered here shows a different behavior for increasing $K$ starting from a value below $K_{c}$. We observe a transition from a state where topological defects are generated continuously by the dynamics to a partial phase synchronized state with moving topological defects, similar to the case with surviving point defects. This partial phase synchronized state is characterized by a large number of phase synchronized oscillators and a 


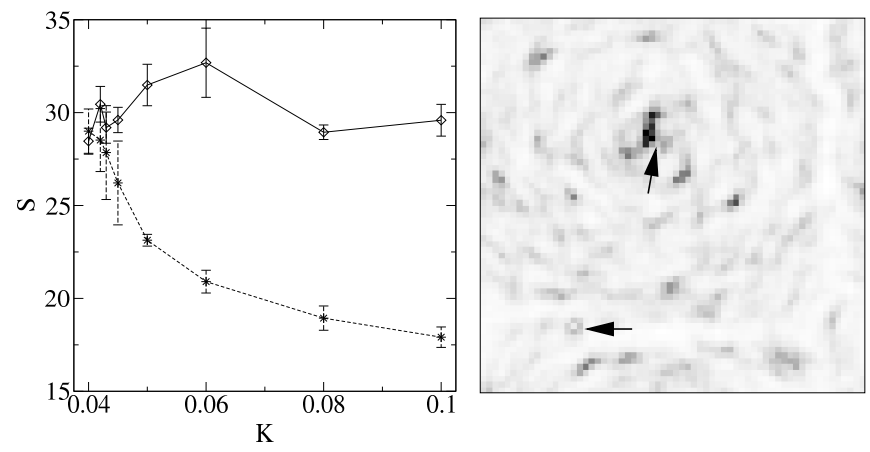

FIG. 5: Left: $S$ as a function of $K$ for homogeneous (star) and inhomogeneous (diamonds) initial conditions. The results were obtained from an average over five realizations of the random $\omega(\mathbf{r})$ field. The phase coherence is higher when (more) topological defects are present in the system for $K>K_{c} \approx$ 0.043. Below $K_{c}$ point defects are created and annihilated dynamically. Right: $S(\mathbf{r})$ for the same $\omega(\mathbf{r})$ realization as in Figs. 3 and 4 . Dark areas correspond to high values of $S(\mathbf{r})$. The locations of the defects are given by the two arrows. The lower left defect is the moving defect. small number of oscillators close to the point defects with mutually different frequencies. Thus, only one cluster with more than one oscillator exists in contrast to the chain geometry. The existence of topological defects in two dimensions and higher dimensions suggests that the transition described here should be prevalent for general networks of chaotic oscillators.

We have shown that the occurrence of topological defects in two-dimensional arrays of nonidentical chaotic oscillators has a two-fold effect: moving topological defects lead to a breakup of global phase synchronization, while all topological defects - especially quasi-stationary ones - increase the phase coherence of the system significantly. Since moving and quasi-stationary topological defects are present in many circumstances, the apparently contradictory scenario of increased phase coherence but loss of global phase synchronization occurs.

\section{Acknowledgments}

This work was supported in part by a grant from the Natural Sciences and Engineering Research Council of Canada. We thank G. Rousseau for providing the numerical integrator.
[1] A. S. Pikovsky, M. Rosenblum, and J. Kurths, Synchronization: A universal concept in nonlinear science (Cambridge University Press, Cambridge, UK, 2001).

[2] S. H. Strogatz, Physica D 143, 1 (2000).

[3] Y. Kuramoto, Chemical oscillations, waves, and turbulence (Springer, Berlin, Germany, 1984).

[4] A. S. Pikovsky, M. G. Rosenblum, G. V. Osipov, and J. Kurths, Physica D 104, 219 (1997).

[5] K. Josić and D. J. Mar, Phys. Rev. E 64, 056234 (2001).

[6] M. Rosenblum, A. S. Pikovsky, and J. Kurths, Phys. Rev. Lett. 76, 1804 (1996).

[7] R. C. Elson, A. I. Selverston, R. Huerta, N. F. Rulkov, M. I. Rabinovich, and H. D. I. Abarbanel, Phys. Rev. Lett. 81, 5692 (1998).

[8] V. Makarenko and R. Llinás, Proc. Nat. Acad. Sci. USA 95, 15474 (1998).

[9] C. Schäfer, M. G. Rosenblum, J. Kurths, and H. H. Abel, Nature 392, 239 (1998).

[10] I. Z. Kiss, Y. Zhai, and J. L. Hudson, Phys. Rev. Lett. 88, 238301 (2002).

[11] B. Blasius, A. Hupert, and L. Stone, Nature 399, 354 (1999).

[12] P. Tass, M. G. Rosenblum, J. Weule, J. Kurths, A. S. Pikovsky, J. Volkmann, A. Schnitzler, and H.-J. Freund, Phys. Rev. Lett. 81, 3291 (1998).

[13] R. Fitzgerald, Physics Today 52, 17 (1999).

[14] A. S. Pikovsky, M. G. Rosenblum, and J. Kurths, Europhys. Lett. 34, 165 (1996).

[15] Z. Liu, Y.-C. Lai, and F. Hoppensteadt, Phys. Rev. E 63, 055201 (2001).

[16] Y. Zhang, G. Hu, and H. A. Cerdeira, Phys. Rev. E 64,
037203 (2001)

[17] D. L. Valladares, S. Boccaletti, F. Feudel, and J. Kurths, Phys. Rev. E 65, 055208 (2002).

[18] Z. Zheng, G. Hu, and B. Hu, Phys. Rev. Lett. 81, 5318 (1998).

[19] R. Kapral and K. Showalter, eds., Chemical waves and patterns (Kluwer Academic Publishers, Dordrecht, Netherlands, 1996).

[20] D. Walgraef, Spatio-temporal pattern formation (Springer, New York, 1997).

[21] A. Mikhailov, Foundations of synergetics I: Distributed active systems (Springer, New York, 1994).

[22] N. Mernin, Rev. Mod. Phys. 51, 591 (1979).

[23] The motion of chaotic oscillators cannot be uniquely parametrized by the phase in contrast to classical oscillators; however, the phase variable suffices for the determination of the location and charge of a topological defect in the spatially-distributed medium.

[24] A. Goryachev, R. Kapral, and H. Chaté, Int. J. Bif. Chaos 10, 1537 (2000).

[25] O. Rössler, Phys. Lett. A 57, 397 (1976).

[26] C. Zhou and J. Kurths, Phys. Rev. E 65, 040101 (2002).

[27] The distortion of the local orbits by the motion of the topological defects cannot only cause the breakdown of global phase synchronization but also the breakdown of a proper phase description, i.e., no unique center of rotation exists. However, the percentage of loops in phase space where $(0,0)$ lies outside the loop is of the order of $0.1 \%$ for $K$ close to $K_{0}$ and, thus, extremely small. In particular, the effect on the estimate of the oscillator's frequency is negligible. 
[28] I. S. Aranson and L. Kramer, Rev. Mod. Phys. 74, 99 (2002).

[29] A. Goryachev and R. Kapral, Phys. Rev. Lett. 76, 1619 (1996).

[30] These scenarios include, for example, a non-monotonical dependence of the number of topological defects on $K$ and fluctuations in the number of topological defects on very short time scales.

[31] P. Coullet, L. Gil, and J. Lega, Phys. Rev. Lett. 62, 161 (1989). 